\title{
COMPRENSIÓN LECTORA Y RENDIMIENTO ESCOLAR
}

\section{READING AND LOW SCHOOL PERFORMANCE COMPREHENSION}

\author{
Consuelo de los Ángeles Molina Ibarra ${ }^{1}$ \\ Pontificia Universidad Católica del Ecuador. \\ Cotopaxi-Ecuador.
}

\section{RESUMEN}

Una herramienta vital dentro del campo educativo, para acceder al conocimiento y la construcción de la realidad, es la comprensión lectora. Esta representa un elemento significativo en el desarrollo del ser humano. El objetivo de esta investigación es determinar cómo la comprensión lectora influye en el rendimiento escolar de los estudiantes de séptimo grado de la Unidad Educativa "Manuelita Sáenz". Se ha podido constatar que la problemática de la comprensión lectora es un tema que se presenta en la literatura internacional como una de las principales dificultades que propician un bajo rendimiento escolar. Diferentes investigaciones realizadas en Chile, Perú, España, Mé-

Pontificia Universidad Católica del Ecuador.

Cotopaxi-Ecuador.

Orcid:

https://orcid.org/0000-0003-0531-6023 xico, destacan la interrelación entre estos dos aspectos y consideran que un factor importante para lograr un alto rendimiento académico, es que los estudiantes sean capaces de extraer información, de interpretar lo leído e inferir el significado, así como evaluar críticamente el contenido del texto, lo cual aumentaría, además, el vocabulario y por ende su aprendizaje significativo. Se puede constatar en varios autores. Para determinar el nivel de comprensión lectora, en los 15 estudiantes de séptimo grado de la Unidad Educativa Manuelita Sáenz, de la provincia de Cotopaxi, Cantón Latacunga Ecuador, se le aplicó un test basado en diferentes indicadores seleccionados por la autora donde se median a través de los contenidos más complejos abordados en la asignatura de Lengua y Literatura. La investigación fue el tipo descriptiva explicativa. Se corroboró que existe una estrecha relación entre el nivel de comprensión lectora y el bajo rendimiento académico en este grupo de estudiantes. 
PALABRAS CLAVES: comprensión lectora, rendimiento escolar, niveles de lectura.

\section{ABSTRACT}

A vital tool within the educational field, to access knowledge and the construction of reality is reading comprehension. It plays a significant role in human development. The aim of this research es to determine how reading comprehension influences the school performance of seventh grade students in the Manuelita Sáenz Educational Unit. It has been observed that the problem of Reading comprehension that is presented in international literature as one of the main difficulties that lead to low school performance. Different investigations carried out in Chile, Peru, Spain, Mexico, highlight the interrelationship between these two aspects and consider that an important factor to achieve a high academic performance is that student are able to extract information, interpret what they read and infer the meaning, as well as critically evaluate the content of the text, which would also increase the vocabulary and therefore their significant learning. This can be seen in several authors. In order to determine the level of Reading comprehension, an assessment was applied to the 15 seventh grade students of the above-mentioned educational unit. Different indicators selected by the author were used in different contents approached in the subject of Language and Literature. The research that will be applied is of the descriptive explanatory type. It was corroborated that there is a close relationship between the level of reading comprehension and the low academic performance in this group of students.

KEYWORDS: Reading comprehension, school performance, levels of Reading.

\section{INTRODUCCIÓN.}

La lectura no es solamente la reproducción y repetición de la comunicación escrita, sino es entender como fue construido el texto, para ello se necesita manejar un buen número de palabras y saber entrelazarlas sintácticamente.

La lectura es un proceso de comunicación entre el escritor (texto) y el lector (sujeto), donde el escritor (que en este caso significa persona que escribe), transmite mensajes o información al lector (persona que lee), a través de la escritura de la lengua. El proceso de comunicación escrita es bastante complejo, en el cual el escritor "codifica" el mensaje a transmitir al lector y este al leerlo, decodifica dicha información. Este proceso se inicia en el cerebro de quien escribe y termina en el cerebro del que lee. Los signos gráficos que utiliza el escritor para hacer conocer sus mensajes o sus intenciones deben estar previamente almacenados en su cerebro. Estas intenciones se asimilan cuando se desarrolla la habilidad lingüística de leer. En consecuencia, para escribir hay que aprender a leer y desarrollar la lectura continuamente (Cassany, 2014)

La lectura juega un papel importante en la formación cultural de las personas, en el aprendizaje y dominio de la lengua. Según Mayor (2001) la lectura es importante porque favorece el desarrollo del pensamiento ya que estando en ella se reflexiona, medita y se crea. En otro sentido la lectura comprensiva es un mecanismo positivo porque permite el desarrollo del pensamiento crítico, creativo y divergente.

A tono con lo anterior en todos los niveles de enseñanza los docentes deben concientizar la importancia que tiene formar esta habilidad, siendo ella una de las competencias que se requieren tanto para la vida como para ejercer cualquier profesión.

\section{ESTADO DEL ARTE}

Para entender el significado de leer como comprender señala Palacios (2001), citado por Gómez: 
"El significado de un texto no reside en la suma de significados de las palabras que lo componen, sino solo coincide con el significado literal del texto ya que ellos se construyen los unos en relación con los otros" (Gomez, 2011, pág. 30).

La aceptación del significado de cada palabra depende de la frase donde aparece; así como el párrafo puede contener la idea central de un texto o construir un simple ejemplo según su articulación en el discurso. Un mensaje verbal jamás ofrece la totalidad de la información, sino que el emisor lo construyó simplemente con la información que juzgó necesaria para que el receptor la entienda, suponiendo que hay muchas cosas que no hay que explicitar. Para Valladares (1998), la comprensión de textos está presente en todos los escenarios de todos los niveles educativos y se considera una actividad crucial para el aprendizaje escolar; todo lo que los alumnos adquieren, discuten y utilizan en las aulas surge a partir de los textos escritos (Gomez, 2011, pág. 30).

Durante mucho tiempo, sin embargo, esta actividad fue relegada en la educación por centrarse demasiado en la enseñanza de habilidades simples de decodificación y automatización de la lectura; actividades asociadas con la interpretación parcial e inadecuada del concepto de alfabetización.

La comprensión de textos es una actividad constructiva compleja de carácter estratégico que implica las interacciones de características del lector y del texto, dentro de un contexto determinado.

Garner y Reis (1981: cit. Por Díaz Barriga y Hernández, 2002) señalan que muchos alumnos no pueden darse cuenta de la existencia de muchos obstáculos que pueden encontrarse cuando leen los textos, por lo tanto no pueden prever las medidas necesarias para autorregular su proceso de comprensión o bien de auto-supervisión. Burón (1993) Díaz Barriga y Hernández, 2002). Denomina a este fenómeno metaignorancia, ya que el lector no se da cuenta de la existencia de obstáculos que hay que regular, lo cual implica no saber diferenciar cuando se está comprendiendo y cuando no. cit. (Gomez, 2011, pág. 31).

El plan para leer el texto debe elaborarse teniendo en cuenta las variables metacognitivas de la persona, tareas y estrategias, en base a esto se pueden seleccionar las estrategias con mayor pertinencia para desarrollar la tarea de lectura. (Barriga, 2014). Nos dice que los lectores eficientes son quienes saben decidir cuales estrategias de las que conocen son las que favorecen el rendimiento en la comprensión en función de los propósitos planteados al inicio de la lectura.

En todo este trabajo de leer es importante tomar encuentra el aprendizaje previo, que el ser humano posee. Según (Artega, 2011) La comprensión de textos tiene como antesala el acto de leer; en este sentido leer es un proceso intelectual y cognitivo que nos permite obtener información a partir de un texto.

La comprensión es producto de una serie de acciones, atender y entender las explicaciones en clase, identificar y relacionar los conceptos claves, organizar la información relevante para dar lugar a que las ideas se conviertan en nuevos conocimientos. Es en la medida que se ejercite la comprensión en la que gradualmente se agiliza y se vuelve habilidosa. (Barriga, 2014)

\section{NIVELES DE COMPRENSIÓN LECTORA}

Los niveles de la comprensión lectora se entienden como métodos de pensamiento que tienen lugar en el proceso de la lectura, los cuales van interviniendo paulatinamente a medida que quien lee utiliza sus conocimientos previos y los asocia a la lectura. 
Para realizar este proceso es necesario citar los niveles existentes.

El primero de ellos es el nivel Literal, el segundo es el nivel inferencial, y el tercero es el nivel crítico ${ }^{2}$.

\section{NIVEL DE COMPRENSIÓN LITERAL}

En esta etapa se lee conforme al texto, en este nivel se solicita respuestas simples, que están explicitas en la lectura, pero requiere que conozcas las palabras. Podríamos dividir este nivel en dos: nivel primario y nivel de profundidad.

La lectura de nivel primario, se centra en las ideas e información que están explícitamente expuestas en el texto, por reconocimiento o evocación de los hechos. El reconocimiento puede ser el detalle, que es el que identifica nombres, personajes, tiempo y lugar de un relato o del texto, la idea más importante de un párrafo, identifica el orden de las acciones.

Realiza una lectura elemental, paso a paso del texto, lo situamos en una determinada época, lugar, e identificamos personajes principales y secundarios.

Muchos de los fracasos escolares en la lectura son por la falta de conocimiento del vocabulario,

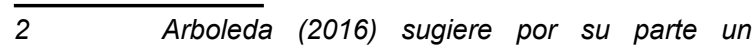
cuarto nivel, quizás el más complejo: el nivel edificador, interrelaciona con los anteriores aunque con diferencia especifica. Aboga por una comprensión edificadora, considerando que la comprensión no puede reducirse a un sentido ni axiológico ni semántico cognitivo, sino que, aunque las incorpora, va más allá de estas dos dimensiones. "El nivel edificador subsume niveles reconocidos en el proceso de la lectura comprensiva y precisa del sujeto la capacidad de dilucidar pasivos frente al acto de vivir y generar creativa, crítica y operativamente acciones para alumbrar vida" (...). Este nivel, en virtud del procesamiento en referencia, convierte la lectura y producción textual comprensiva en procesos fácticos, acontecimentales en donde comprender auténticamente es posible si se incluye en tal mecanismo acciones edificantes de hecho, heurísticamente humanizantes, experiencias urdidas en el relato de su propia comprensión y crecimiento personal y social. Si da lugar, por ejemplo, a que el comprendedor relate su práctica de participación en la construcción de ámbitos de vida que dignifiquen la condición humana. por la falta de conocimiento en las expresiones metafóricas.

Lectura literal en un nivel primario (nivel 1)

Se centra en la idea e información que están explícitamente expuestas en el texto por reconocimiento o evocación de los hechos. El reconocimiento consiste en la localización e identificación de los elementos del texto. (Gordillo, 2009). Que pueden ser:

1.- de ideas principales: Es decir la idea más importante de un párrafo o relato;

2.- de secuencias: identifica el orden de las acciones:

3.- por comparación; identifica caracteres, tiempos y lugares explícitos.

4.- De causa o efecto; identifica razones explicitas de ciertos sucesos o acciones.

\section{NIVEL DE COMPRENSIÓN INFERENCIAL}

En este nivel se describe por indagar y darse cuenta de la relación y asociación de significados que permiten al lector leer entre líneas y entender o deducir la idea implícita. En este nivel el lector explica lo leído más ampliamente, es capaz de agregar informaciones y experiencias anteriores, utilizando los conocimientos previos con los conocimientos nuevos, para así afianzar lo leído y hacerlo propio y significativo. La meta del nivel inferencial, es la elaboración de conclusiones. Este segundo nivel de comprensión es muy poco desarrollo por el lector, ya que requiere un grado de abstracción. El concepto de inferencia comprende, tanto las deducciones estrictamente lógicas, como las conjeturas o suposiciones que pueden realizarse a partir de la lectura misma.

En este nivel se encierra las siguientes operaciones.

1.- Inferir detalles adicionales que, según las 
conjeturas del lector, pudieron haberse incluido en el texto para hacer lo más informativo, interesante y convincente:

2.- Inferir ideas principales, no incluidas explícitamente.

3.- Inferir secuencias sobre acciones que pudieron haber sucedido, si el texto hubiera terminado de otra manera.

4.- Predecir acontecimientos sobre la base de una lectura inconclusa.

5.- Interpretar un lenguaje figurativo, para inferir la significación de un texto.

\section{NIVEL DE COMPRENSIÓN CRÍTICO}

A este nivel se le considera el ideal, ya que en él lector es capaz de emitir juicios de valor, sobre el texto leído, aceptarlo o rechazarlo, pero con argumentos. La lectura crítica tiene un carácter evaluativo, en el que interviene la formación del lector, su criterio y conocimiento de lo leído.

Dichos juicios toman en cuenta cualidades de veracidad, aceptabilidad, probabilidad y los juicios de valor pueden ser:

1.- de realidad o fantasía: según la experiencia del lector, con las cosas que lo rodean o con los relatos de las lecturas.

2.- De adecuación y validez: compara lo que está escrito con otras fuentes de información.

3.- De apropiación: requiere de evaluación relativa en las diferentes partes para asimilarlo.

4.- De rechazo o aceptación: depende del código moral y del sistema de valores del lector.

Los bajos niveles de comprensión lectora en los estudiantes han traído mayores índices de disertación, baja calidad académica.

Con el propósito de responder a la pregunta de investigación planteada y el objetivo principal, fue necesario asumir una metodología de investigación orientada

\section{RENDIMIENTO ESCOLAR}

El rendimiento académico es un término complejo, pues como se puede ver ya en su conceptualización, se le atribuyen denominaciones varias, tales como aptitud escolar, desempeño académico, rendimiento escolar lo cual destaca como componentes de la definición la aptitud y el esfuerzo del estudiante (Navarro, 2003). Así mismo la complejidad se halla en la diversidad de factores que se le atribuyen. Jiménez (2000) citado por (Navarro, 2003), por ejemplo, la hace depender de la evaluación que comprueba un determinado nivel de conocimientos.

Con el desarrollo de la psicopedagogía se ha profundizado en el valor de los componentes cognitivos del aprendizaje tales como la inteligencia, la motivación, el autocontrol y las habilidades sociales que ahora fungen también como factores del rendimiento (Navarro, 2003). Además de los aspectos ya mencionados y que se podrían categorizar como personales, se estudian también factores sociales e institucionales (Garbanzo, 2007, págs. 43-63). Existen trabajos como el de Mirete, B \& García, F. (2014) que relacionan el rendimiento académico con el uso de las Tics y las redes sociales (Minarete \& García, 2014).

Habría que decir entonces que el rendimiento escolar es más bien un constructor, es decir, una construcción teórica que involucra muchos factores, y que sirve de soporte intelectual para avanzar en la investigación pedagógica. Esto significa para nuestro tema que son variadas las causas del rendimiento escolar y que si se quiere hacer un estudio exhaustivo es necesario realizar mediciones de todos los factores. Sin embargo, como se desprende del estudio de Navarro (2003) tres son los factores 
que tradicionalmente se consideran más importantes, a saber, la aptitud intelectual, el hábito del estudio (el esfuerzo) y las calificaciones obtenidas. Así se puede colegir de trabajos de investigación como el de Martínez (1996) que señala estos como los más destacados, teniendo en cuenta también la personalidad, el clima familiar y pedagógico (Martínez, 1996). Elegimos para el presente trabajo la siguiente definición: "Nivel de conocimientos demostrado en un área o materia comparado con la norma de edad y nivel académico" (Jiménez, 2000) citado por Navarro" (2003).

\section{INFLUENCIA A NIVEL INTERNACIONAL}

Las analogías y no menos divergencias, del ámbito político a nivel mundial, dan cuenta que el tema educativo es prioridad en el mundo.

Por consecuencia, al analizar comparativamente el rendimiento entre ellas no puede ser homogénea, ya que no es posible tomar referencias unánimes y conformes a criterios. Como posibles guías o parámetros que permitan adquirir algunas ideas

\section{Alumnos que no obtienen el título o diploma en educación secundaria}

Obligatoria (Va). Año 1996

\begin{tabular}{|l|l|l|l|l|l|l|l|l|l|l|}
\hline Primaria & Belg. & Alema. & Grecia & Españ & Fran & Irlan & Lux & Itali & P.Bajo. & ReinU. \\
\hline & 89 & 8 & 11 & 28 & 18 & 9 & 7 & 10 & 16 & 9 \\
\hline
\end{tabular}

Fuente: Eurostat, U.O.E. Eurydice. Reestructurado por la autora

\section{INFLUENCIA A NIVEL NACIONAL.}

El Ecuador ha perdido el año en calidad de la educación. Los informes nacionales e internacionales así lo expresan. La calidad, como todos sabemos, está asociada a la formación y capacitación de los docentes. En este sentido, la política del Sí Profe ha fallado, porque sus
Comparativas, se presentan algunos datos: Gastos por alumnos de establecimientos públicos de enseñanza, primaria y secundaria obligatoria, en miles de euros por alumno (1996)

Bélgica: Comunidad flamenca únicamente, Los datos de la enseñanza secundaria inferior agrupan los niveles en inferior y superior. Grecia y Luxemburgo: Los datos de la enseñanza primaria agrupan la educación preescolar y la primaria y los de la enseñanza secundaria inferior agrupa también la superior. España: Los datos de la enseñanza secundaria inferior agrupan los niveles obligatorios y Bachillerato. Irlanda: La mayoría de los colegios son privados concertados aunque los datos fueron dados como si fueran de enseñanza pública. Países bajos: Las escuelas públicas son minoritarias, aunque la ley dc 1917 prohíbe tratar diferenciadamente los dos sectores. Reino Unido: Los datos de enseñanza secundaria inferior agrupan los niveles obligatorios y superiores. Islandia: Los datos de la enseñanza primaria agrupan la educación preescolar y la primaria y los de la enseñanza secundaria obligatoria agrupan también la superior. Noruega: Los datos no se proporcionaron de forma desglosada. (Cano, 2001) 
cativas, en los sectores urbano-marginales y rurales. Existe un pobre desarrollo de los procesos pedagógicos, una ausencia de infraestructura y un personal poco preparado.

\section{METODOLOGÍA.}

La metodología plantea una forma práctica de llevar a cabo, o lograr la finalidad que se propone. En el aprendizaje significativo la finalidad es de lograr que el nuevo conocimiento tenga significados para el estudiante al reaccionarlos con conocimientos anteriores, por lo cual se puede aplicar la siguiente metodología.

Los investigadores han indicado a lo largo del tiempo que la lectura se puede enseñar en fases para mejorar la comprensión.

Este estudio profundiza en comprender como la situación en el aula y los métodos utilizados por los maestros de enseñanza utilizados en la Unidad

\section{ENFOQUE}

Entre las características de esta investigación están: un enfoque cualitativo, ya que se utiliza una recolección de datos no numérica, que permite generar preguntas a lo largo de la investigación, para refinarlas y responderlas. A medida que se va averiguando los hechos se los va interpretando. El estudio puede ser modificado dependiendo los factores que rodean al mismo. Se realizó la muestra con el fin de realizar un diagnóstico de la situación de los estudiantes, luego de la recolección se analizaron los resultados mediante la metodología utilizada por el programa SPS, finalmente se interpretó los datos, contrastando la correlación entre comprensión lectora y rendimiento, se obtuvo como resultado que existe una estrecha relación entre ambas variables.

(Ries, 1981) Citado por Diaz Barriga Hernández, 2002) "señalan que muchos alumnos no pueden darse cuenta de la existencia de muchos obstáculos que pueden prever las medidas necesarias para autorregular su proceso de comprensión o bien de autosupervisión, denomina a este fenómeno meta ignorancia, ya que el lector no se da cuenta de la existencia de obstáculos que hay que regular, lo cual implica no saber diferenciar cuando se está comprendiendo algo o no".

Se lo realiza de manera simultánea. Se obtiene las perspectivas y puntos de vista de los participantes (sus emociones, prioridades, experiencias, significados y otros aspectos subjetivos). Y finalmente se evalúa el desarrollo natural de los sucesos.

El diseño de la investigación es de una teoría fundamentada que significa que la teoría (hallazgos) va emergiendo fundamentada en los datos, con la finalidad de explorar y describir, y luego generar perspectivas teóricas.

\section{TÉCNICAS DE RECOLECCIÓN}

La técnica que se utilizó fue la observación con un test de registro en la cual se observó cada una de las clases, aplicándose en el área de lengua y literatura el test permitió darnos cuenta que los estudiantes de 7 mo grado, no tienen el hábito de leer; este test fue procesado a través del programa SPS.

El alcance de esta investigación es descriptiva explicativa, y la población que se utilizó son 15 estudiantes de séptimo año de educación general básica de la escuela antes mencionada.

Se eligió el estudio de las dos variables independiente y dependiente, teniéndose como variable independiente la comprensión lectora, y como variable dependiente el rendimiento académico, se recurrió a el tipo de investigación cualitativa ordinal en la variable independiente, y en la otra variable dependiente, cuantifica, lo cual quiere decir que la investigación es mixta, 
considerando la escala Likert para el estudio de los datos investigados, una vez ingresados los datos se analizó la fiabilidad utilizando el Alpha de Cronbach, obteniéndose como validez del constructo KMO. En cuanto a la validez de contenido se estableció una correlación de ítems total.

En la estadística descriptiva se trabajó, con las medidas de tendencia central que son moda y mediana para la variable independiente, con la mediana, y media aritmética para la variable dependiente. También se trabajó con las medidas de dispersión con la variable dependiente con la varianza

\section{RESULTADOS}

La lectura, en efecto constituye un proceso estratégico de adquisición de significado que necesita la conciencia de los muchos procesos de razonamiento implicados y controlados, ya que cabe señalar que en la lingüística social o geográfica se empleara el mismo sistema lingüístico de manera más o menos diferenciada, según las circunstancias más diversas y según el contexto comunicativo.

De hecho, (Tonelotto, 2005-2008) han demostrado que tanto las habilidades de reconocimiento de las palabras como la comprensión de la lectura están relacionadas con el rendimiento académico, indicando de esta manera que las habilidades de la lectura son buenos predictores del rendimiento escolar.

Para ello y por medio de las aplicaciones de test de comprensión lectora, se verificó que en los niños; se prestó atención al desarrollo del concepto de la lectura en los alumnos del Séptimo grado. Para lo cual el $30 \%$ de los estudiantes nos refleja que la lectura genera el desarrollo del intelecto. Conciben la comprensión como producto de una serie de acciones, atender y entender las explicaciones en clase, identificar y relacionar los conceptos claves, organizar la información relevante para dar lugar a que as ideas se conviertan en nuevos conocimientos y estar en condiciones de entregar productos de lectura. A pesar que no especificaron que la lectura es la base del aprendizaje se puede inferir que al exponer que el intelecto se fomenta con la lectura están considerando que una lectura eficiente sustenta el aprendizaje.

El $70 \%$ de alumnos del séptimo grado deja claro que la lectura eleva el conocimiento, mejora el dominio del lenguaje, ayuda a manejar mayor la cantidad de términos y se mejora la dicción. Como lo explica (Smith, 1989) el desarrollo del vocabulario es consecuencia de la capacidad cognitiva en base a la comprensión lectora la capacidad verbal aumenta. Sin embargo, como se había dicho que tienen relación las dos variables tanto la de la comprensión lectora, como la del rendimiento escolar, con los resultados obtenidos podemos decir que si existe relación íntimamente ligada a esta hipótesis. Cabe señalar también que la lectura es una habilidad compleja cuya finalidad última persigue la comprensión de un texto escrito. Esta ampliamente consensuado que los dos grandes componentes de la lectura son el reconocimiento de las palabras y la compresión lectora.

\section{CONCLUSIONES}

Se puede concluir diciendo que la comprensión lectora incide en el aprendizaje significativo al tratarse de una destreza básica para el entendimiento de los contenidos, no obstante mientras el alumno está en la escuela tiene la asistencia del profesor quien se encarga generalmente de explicar cualquier contenido disminuyendo el impacto que la baja comprensión lectora puede tener. Sin embargo en casa el menor puede sufrir las consecuencias de una baja comprensión lectora si no cuenta que despeje sus dudas. Es de tal manera que la comprensión lectora puede afectar el aprendizaje de varias materias en general: sin embargo, 
por lo antes expuesto, el impacto de la misma se ve disminuido.

La baja comprensión lectora a futuro puede acarrear más problemas de los que se pueden presentar a nivel escolar, el alumno por ende puede generar fallas de redacción, poco vocabulario, bajo nivel de auto aprendizaje, poco desarrollo del pensamiento análogo, y poca atención en los contextos y demasiada atención en las palabras.

Entre los factores detectados que afectan la comprensión lectora en la muestra investigada, se encuentra principalmente la falta de hábitos de lectura, por lo que no se promueve la costumbre de leer voluntariamente. El objetivo de la investigación es también dar pautas o instrumentos de intervención que tengan los actores, del proceso enseñanza aprendizaje.

Son factores también la falta de interés por el tema de las lecturas, una técnica poco adecuada para leer y el poco apoyo o motivación por parte de los adultos que rodean a los estudiantes (padres de familia, sociedad en la se desenvuelven y docentes) es así como la comprensión lectora se ve afectada por la motivación para empezar a leer, sin embargo, si un estudiante motivado empieza a leer pero no entiende, la motivación inicial se perderá, de forma que la comprensión lectora y la motivación para hacerlo están relacionadas entre sí.

La técnica de la lectura utilizada por el estudiante también influye en la facilidad o dificultad para comprender el texto, de forma que los estudiantes que no han superado el estilo de lectura manejado durante el aprendizaje de la misma (en voz alta y palabra por palabra).

Se presenta en el siguiente cuadro el desarrollo de la investigación con los resultados obtenidos en la misma.

\begin{tabular}{|l|l|l|}
\hline $\begin{array}{l}\text { PRUEBAS } \\
\text { ESTADISTICAS }\end{array}$ & VALOR & ANALISIS \\
\hline Alfa de conbach &, 845 & $\begin{array}{l}\text { Esta prueba relaciona los ítems de } \\
\text { instrumento, cuyo valor para ser aceptado } \\
\text { debe ser mayor 0,7, por ende el valor } \\
\text { obtenido es fiable y se puede utilizar el } \\
\text { instrumento. }\end{array}$ \\
\hline $\begin{array}{l}\text { Medidas de tendencia } \\
\text { central }\end{array}$ & Moda: & $\begin{array}{l}\text { Calcula la mayor frecuencia de los datos } \\
\text { distribuidos. }\end{array}$ \\
\hline Mediana: & $\begin{array}{l}\text { Representa el valor de la variable de posición } \\
\text { central en un conjunto de datos ordenados. }\end{array}$ \\
\hline
\end{tabular}




\begin{tabular}{|c|c|c|}
\hline Tabla de frecuencia. & $\begin{array}{l}\text { Frecuencia absoluta } \\
\text { Frecuencia Relativa } \\
\text { Frecuencia } \\
\text { acumulada } \\
\text { Frecuencia relativa } \\
\text { acumulada }\end{array}$ & $\begin{array}{l}\text { Frecuencias absolutas para todos los eventos } \\
\text { iguales o anteriores que un cierto valor. } \\
\text { La frecuencia relativa se puede expresar en } \\
\text { tantos por ciento y se representa por } n \\
\text { Es la frecuencia absoluta acumulada y el total } \\
\text { de la muestra. } \\
\text { Es el cociente entre la frecuencia acumulada } \\
\text { de un determinado valor y el número total de } \\
\text { datos. }\end{array}$ \\
\hline Medidas de dispersión & Varianza & $\begin{array}{l}\text { Relaciona la variabilidad de los resultados } \\
\text { entre sí de cada ítem; como es el caso de la } \\
\text { pregunta } \mathbf{N}^{\circ} \mathbf{2} \text { es de }, 314 \text { es decir no existe } \\
\text { mayor variabilidad en las respuestas. }\end{array}$ \\
\hline Estadística Inferencial & 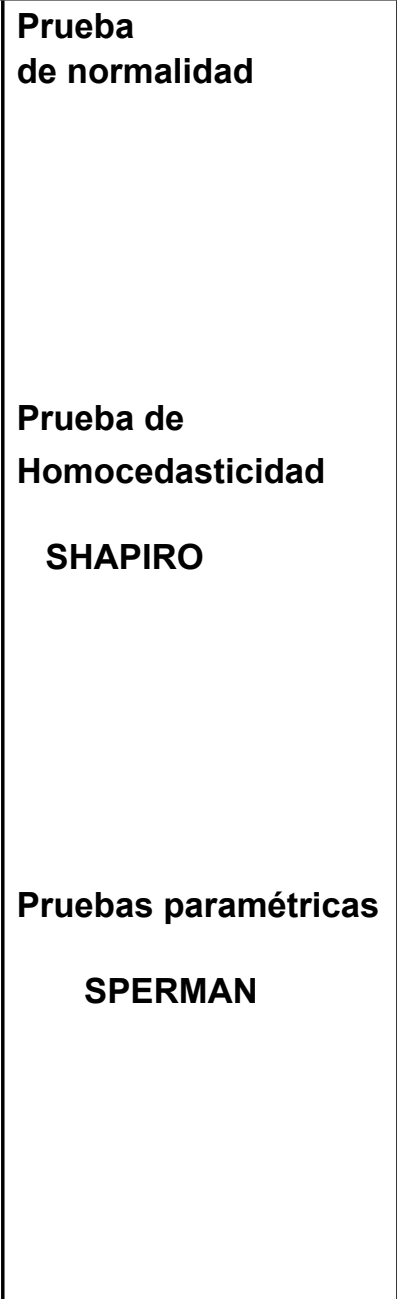 & $\begin{array}{l}\text { Son los resultados de la prueba indican si se } \\
\text { rechazan o no puede rechazar la hipótesis } \\
\text { nula de que los datos. } \\
\text { Para los resultados se obtiene valores } \\
\text { menores a } 0,5 \text { no tiene una distribución } \\
\text { normal por ende se realiza pruebas no } \\
\text { paramétricas. } \\
\text { Se utiliza para evaluar la igualdad de las } \\
\text { varianzas para una variable. } \\
\text {,892 en su mayoría son iguales, es decir } \\
\text { tienen igualdad. } \\
\text { Se aplica esta prueba debido a que los datos } \\
\text { son correlacionales y descriptivos } \\
\text { Según refleja el instrumento de evaluación } \\
\text { utilizado. } \\
\text { En esta prueba se relaciona cada uno de } \\
\text { los ítems utilizado en el instrumento, y el } \\
\text { programa SPSS, marca (con un asterisco) los } \\
\text { ítems que tiene relación significativa al } 0,05 \\
\text { y con (dos asteriscos)a los que se relacionan } \\
\text { significativamente al } 0.01\end{array}$ \\
\hline
\end{tabular}




\section{ANALISIS DE DATOS.-}

En esta investigación lo que se quiere es determinar cómo la comprensión lectora influye en el rendimiento escolar de los estudiantes de séptimo grado de la Unidad Educativa "Manuelita Sáenz; Para lo cual se procedió a verificar la problemática planteada que es la falta de comprensión lectora, en las lecturas que los niños desarrollan, por tal motivo era importante realizar la operacionalización de variables se utilizó como fuente de investigación el artículo sobre el rendimiento escolar (Lamas, 2015).

Aplicando para la investigación el instrumento de evaluación se obtuvo el 0,845 en el alfa de combrach, el mismo que es un valor aceptable para sustentar un instrumento de análisis fiable, para que este estudio sea aceptado debe ser mayor a 0,7 por ende el valor obtenido nos muestra que si podemos utilizar este instrumento; también trabajamos con las medidas de tendencia central como es la moda y la mediana, ya que nos permitieron calcular la mayor frecuencia de los datos distribuidos; también trabajamos con la tabla de frecuencia absoluta, y frecuencia acumulada ya que estas nos permiten tener un valor consiente y un numero de datos exacto; se utilizó las medidas de dispersión como es la Varianza ya que esta relaciona la variabilidad de los resultados entre sí de cada ítem; como es el caso de la pregunta $N^{\circ} 2$ que es de 0,314 es decir no existe mayor variabilidad en las respuestas:

\section{REFERENCIAS BIBLOGRÁFICAS}

Arboleda, J. C. (2016). Elaboración de textos académicos en el marco de la comprensión crítico edificadora. Revista Boletín Redipe, 8/7.

Artega, L. (2011). la comprension lectotra y el bajo rendimiento escolar. Revista de comunicacion e investigacion een desarrollo, 27-36.

Barriga, D. (2014). la comprension lectora. Ra
Ximhai, 265-277.

Cano, J. (2001). El rendimietno escolar y sus contextos. Revista Complutense de Educacion, 15-80.

Cassany, L. (2014). La comprension lectora. Revista cientifica de america latina.

Garbanzo, G. (2007). Factores asociados al rendimiento académico en estudiantes universitarios, una reflexión desde la calidad de la educación superior pública. Revista Educación, 43-67.

Gomez, J. (2011). Comprension Lectora y Escolar, Una ruta para mejorar la comunicacion. COMUNI@CCIÓN: Revista de Investigación en Comunicación y Desarrollo, 27-36.

Gordillo, A. D. (2009). Los niveles de comprension lectora. Revista Actualidades Pedagogicas $N^{\circ} 53$, 1- 14.

Lamas, H. (2015). Sobre el rendimiento escolar. Propositos y representaciones, 313-386.

Martínez, V. (1996). Factores determinantes del rendimiento académico en Enseñanza Media. Publicación oficial del Colegio de Psicólogos de Madrid, 3-14.

Minarete, B., \& García, F. (2014). Rendimiento Académico y Tic. Una experiencia con webs didácticas en la Univerdiad de Murcia. Pixel-bit. Revista de medios y Educación, 169-183.

Navarro, R. (2003). EL RENDIMIENTO ACADÉMICO: CONCEPTO, INVESTIGACIÓN Y DESARROLLO. REICE - Revista Electrónica Iberoamericana sobre Calidad, Eficacia y Cambio en Educación, 1-13.

Ries, G. y. (1981). Comprension Lectora.

Smith, V. (1989). La Ciencia del Texto. Paidos.

Zamora, E. (01 de 06 de 2013). Desface entre ek rendimiento academico reale ideal y sus efectos en la calidad educativa e los estudiantes. Universidad Tecnca de Babahoyo. Babahoyo, Babahoyo, Ecuador: Publicaciones CEPEC. 